\title{
Pharmaindustrie ist auch für Gesetz gegen Korruption
}

\author{
Die Sensibilität der Pharmaindus- \\ trie für das Thema Korruption \\ nimmt zu. Im Vergleich mit an- \\ deren Branchen sind firmeninter- \\ ne Bemühungen zur Prävention \\ aber noch unterrepräsentiert.
}

Der Zeitpunkt hätte kaum besser gewählt werden können: Just zum Launch einer Gesetzesinitiative, mit der Bestechung und Bestechlichkeit im Gesundheitswesen unter Strafe gestellt werden sollen, veröffentlicht das Beratungsunternehmen PricewaterhouseCoopers (PWC) eine Studie zur Wirtschaftskriminalität in der Pharmaindustrie.

\section{Wachsende öffentliche Diskussion}

Im Fokus stand „das Risiko, in geschäftlichen Beziehungen zu Ärzten mit Antikorruptionsregelungen in Konflikt zu geraten“.

Offenkundig, das zeigt die PWC-Erhebung, hat die wachsende öffentliche Diskussion über Korruption sowie der verbreitete Trend $\mathrm{zu}$ intensiver Beschäftigung mit Compliance auch bei den Arzneimittelherstellern das Bewusstsein für fragwürdige Marketingpraktiken gegenüber Ärzten geschärft.
So werden etwa Sponsoring, Studienvergabe, Anwendungsbeobachtungen oder Beraterbeziehungen von mittlerweile zwei Dritteln der befragten Firmen als potenziell problembehaftet eingestuft, während dies bei einer ähnlichen Befragung vor zwei Jahren erst rund die Hälfte der Unternehmen so sahen.

\section{Wenig Compliance-Programme}

Aber: Trotz geschärfter Wahrnehmung von Korruptionsrisiken ist die Bereitschaft der Firmen, eigene ComplianceProgramme aufzulegen noch relativ gering ausgeprägt.

Branchenübergreifend ermittelte PWC, haben $46 \%$ aller Unternehmen eigene Richtlinien zur Prävention korruptiver Geschäftsbeziehungen verfasst - doch unter den befragten Arzneimittelherstellern nur 22\%. Ein Grund dafür könnte sein, dass an der Studie viele kleine und mittlere Unternehmen teilgenommen hatten, räumt PWC-Partner Steffen Salvenmoser ein. Nicht zuletzt wegen fehlender interner Ressourcen verließen die sich eher auf externe Selbstverpflichtungswerke wie den FSA oder den AKG. (Christoph Winnat)

Ärzte Zeitung, 17.04.2013

\section{Kritische Selbsterkenntnis}

Das Gesundheitswesen, die pharmazeutische Industrie und die Ärzteschaft im besonderen, haben wirklich ein Korruptionsproblem. Das ist nicht das Urteil von Berufskritikern wie Transparency International, sondern die Selbsterkenntnis von Unternehmen der Pharma-Branche.

Eine jetzt veröffentlichte Studie des Wirtschaftsberatungsunternehmens PricewaterhouseCoopers zeigt die wachsende Sensibilität der Arzneimittelhersteller und im Vergleich zu anderen Branchen einen möglichen Rückstand in der Korruptionsprävention im Arbeitsalltag.

Die offene Flanke, die die Unternehmen in der Umfrage eingestehen, betrifft aber auch die niedergelassenen Ärzte als Partner. Die Spitzen des Kammer- und KV-Systems, die als Selbstverwaltung auch Ordnungsfunktion haben, müssten alarmiert sein, wenn gängige In strumente der Partnerschaft zwischen Industrie und Ärzten wie Anwendungsbeobachtungen, Teilnahme an Studien oder Sponsoring als potenziell gefährdend eingestuft werden. Und deshalb der Partner Pharma-Industrie nach dem Strafgesetzbuch ruft. Wachsendes Problembewusstsein, aus dem alltagstaugliche Verhaltensregeln folgen, sind der notwendige, aber auch mühsame Weg, der aus dem Generalverdacht der Bestechung herausführt.

helmut.laschet@springer.com

\section{Rechtsschutz: Beistand auch beim Streit mit Banken}

Verstehen Sie, was es mit "Effekten" und "Grundsätzen der Prospekthaftung" auf sich hat? Müssen Sie nicht, sagt der BGH und urteilt zum Nachteil zweier Rechtsschutzversicherer.

Von ihrer Rechtsschutzversicherung können Ärzte auch rechtlichen Beistand im Streit um Geld- und Kapitalanlagen einfordern. Zwei übliche Klauseln, mit denen die Versicherer dies bislang weitgehend ausgeschlossen haben, sind intransparent und daher unwirksam, wie jetzt der Bundesgerichtshof (BGH) in Karlsru- he entschied. Nach den nun verworfenen Klauseln bestand kein Rechtsschutz „für die Wahrnehmung rechtlicher Interessen in ursächlichem Zusammenhang mit der Anschaffung oder Veräußerung von Effekten (zum Beispiel Anleihen, Aktien, Investmentanteilen) sowie der Beteiligung an Kapitalanlagemodellen, auf welche die Grundsätze der Prospekthaftung anwendbar sind (zum Beispiel Abschreibungsgesellschaften, Immobilienfonds)“.

\section{Verbraucherzentrale klagte}

Aufgrund zahlreicher Beschwerden klagte die Verbraucherzentrale Nordrhein-Westfalen gegen die $\mathrm{R}+\mathrm{V}$ Versicherung und die
WGV-Versicherung und bekam nun vom BGH Recht. Beide Versicherer dürfen die "Effektenklausel“ und die „Prospekthaftungsklausel" nicht mehr anwenden und sich nicht mehr darauf berufen. Zur Begründung erklärten die Karlsruher Richter, die Begriffe „Effekten“ und „Grundsätze der Prospekthaftung" seien nicht ausreichend klar definiert, und erst recht durchschnittliche Verbraucher wüssten nicht, was damit gemeint ist. Daher seien die Ausschlussklauseln intransparent und damit unwirksam.

(mw)

Urteile des Bundesgerichtshofes: Az. IV ZR 84/12 und IV ZR 174/12; Ärzte Zeitung 19.05.2013 